\title{
Mini-open anterior lumbar interbody fusion
}

\author{
Gurpreet S. Gandhoke, MD, Christian Ricks, MD, Zachary Tempel, MD, Brian Zuckerbraun, MD, \\ D. Kojo Hamilton, MD, David O. Okonkwo, MD, PhD, and Adam S. Kanter, MD
}

Department of Neurological Surgery, University of Pittsburgh Medical Center, Pittsburgh, Pennsylvania

In deformity surgery, anterior lumbar interbody fusion provides excellent biomechanical support, creates a broad surface area for arthrodesis, and induces lordosis in the lower lumbar spine. Preoperative MRI, plain radiographs, and, when available, CT scan should be carefully assessed for sacral slope as it relates to pubic symphysis, position of the great vessels (especially at $L 4 / 5$ ), disc space height, or contraindication to an anterior approach. This video demonstrates the steps in an anterior surgical procedure with minimal open exposure.

The video can be found here: https://youtu.be/r3bC4_vu1hQ.

KEYWORDS lumbar spine; sacrum; ALIF; fixation; operative technique; video 